\title{
Surgical Treatment of Lesional Mesial Temporal Lobe Epilepsy
}

Review

Journal of Epilepsy Research pISSN 2233-6249 / eISSN 2233-6257

Received November 25, 2017 Accepted June 22, 2018

Corresponding author:

Seung-Ki Kim, MD, PhD

Division of Pediatric Neurosurgery, Seoul

National University Children's Hospital,

101 Daehak-ro, Jongno-gu, Seoul 03080,

Korea

Tel. $+82-2-2072-308$

Fax. $+82-2-744-8459$

E-mail; nsthomas@snu.ac.kr

\author{
Sangjoon Chong, MD ${ }^{1}$, Ji Hoon Phi, MD, PhD ${ }^{1}$, Ji Yeoun Lee, MD, PhD ${ }^{1,2}$, \\ Seung-Ki Kim, MD, PhD ${ }^{1}$ \\ ${ }^{1}$ Division of Pediatric Neurosurgery, Seoul National University Children's Hospital, Seoul National University \\ College of Medicine, Seoul; ${ }^{2}$ Department of Anatomy, Seoul National University College of Medicine, Seoul, Korea
}

Lesional mesial temporal lobe epilepsy (mTLE) concerns a lesion other than mesial hippocampal sclerosis present in the mesial temporal lobe and causing seizures. The lesions are usually composed of focal cortical dysplasia (FCD) or are tumorous. These are good candidates for surgical treatment. Sometimes, it is difficult to distinguish between tumors and FCD and to determine the extent of required removal. ${ }^{11} \mathrm{C}$-methionine positron emission tomography (PET) is helpful in differentiating lesions before surgery in lesional mTLE. In ${ }^{11} \mathrm{C}$-methionine PET imaging, tumors show a hot uptake, whereas FCD does not. In case of tumorous conditions, the removal of only specific lesions may be considered because the seizure outcome is dependent on complete excision of the tumor. There are several ways to safely access mesial temporal structures. The transsylvian-transcisternal approach is a good way to access the mesial structures while preserving the lateral and basal temporal structures. Actual lesions associated with epileptogenesis in FCD may be larger than they appear on magnetic resonance imaging. For this reason, evaluations to locate sufficient epileptogenic foci, including invasive studies, should be completed for FCD, and epilepsy surgery should be performed according to these results. Regardless, the ultimate goal of all epilepsy surgeries is to maximize seizure control while maintaining neurological function. Therefore, a tailored approach based on the properties of the lesion is needed. (2018;8:6-11)

Key words: Dysembryoplastic neuroepithelial tumor, Epilepsy, Focal cortical dysplasia, Ganglioglioma, Lesionectomy, Mesial temporal lobe

\section{Introduction}

Temporal lobe epilepsy (TLE) is the most common cause of medically intractable seizures. The surgical treatment of TLE has been reported with excellent seizure outcomes by a number of studies ${ }^{1,2}$ and its efficacy and safety was demonstrated by a randomized controlled study. ${ }^{3}$ Based on these studies, standard anterior temporal lobectomy (ATL) with amygdalohippocampectomy (AH) has become one of the standard techniques used in TLE surgery.

Hippocampal sclerosis (HS) is the most common pathologic finding in mesial TLE (mTLE) and is responsible for $65-70 \%$ of surgically mTLE. ${ }^{4}$ In addition, lesions other than HS confined to the mesial temporal lobe (mTL), such as focal cortical dysplasia (FCD), or mixed neuronal and glial tumors (MNGTs), such as dysembryoplastic neuroepithelial tumor (DNT) and ganglioglioma, are also etiologies of mTLE. ${ }^{5}$ Few studies have been conducted on lesional mTLE for etiol- ogies other than $\mathrm{HS}$.

In these lesional mTLEs, especially in the case of tumors, several studies have reported that if the lesion is completely resected, seizure control is satisfactory even though the extent of the resection is more limited than with conventional ATL. ${ }^{6-10}$ This method has also been reported to be advantageous in terms of complications such as hemiparesis, visual field defects, and psychiatric problems that may occur with conventional ATL. ${ }^{11-14}$ The development of microsurgical techniques in recent decades has enabled selective access to deeply located mesial temporal lesions while preserving the lateral temporal lobe (TL). The use of image-guided neuronavigation also allows for a safe approach. ${ }^{15}$ However, FCD often requires more extensive resectioning because the epileptogenic focus could be wider than that seen on magnetic resonance imaging (MRI). ${ }^{16}$ In addition, invasive studies are often needed before removal to determine the epileptogenic focus. The problem is that it is not easy to distinguish tumorous 
conditions and FCD lesions using preoperative imaging modalities, including high-resolution MRI.

Although more evidence is required to determine the significance of limited resection, this approach is attractive in that it complies with the goals of epilepsy surgery to achieve a better seizure outcome with fewer complications and enables tailored treatment. Therefore, the questions of how to perform limited resection before surgery and how to perform the surgery are important and challenging for the epilepsy surgeon.

This review discusses how patients must be evaluated preoperatively, including how the type of lesion can be predicted. A surgical approach for mesial temporal lesions that preserves the lateral $\mathrm{TL}$ is also introduced.

\section{Preoperative evaluation}

Video-electroencephalographic monitoring and high-resolution MRI are indispensable tools in epilepsy surgery; they enable identification and localization of lesions. In addition to the abovementioned tools, imaging modalities, such as positron emission tomography (PET) and single photon emission computed tomography, and neuropsychological tests are required. Sometimes, magnetoencephalography and the Wada test provide additional information. ${ }^{17}$

When verifying a lesion through MRI, it is important to consider the possibility of dual pathology. In particular, several pathological lesions may coexist, such as a tumor with HS or an MNGT with FCD in children. $^{18-21}$ These lesions could all be epileptogenic. The entire TL including the lateral neocortex should be thoroughly evaluated, and
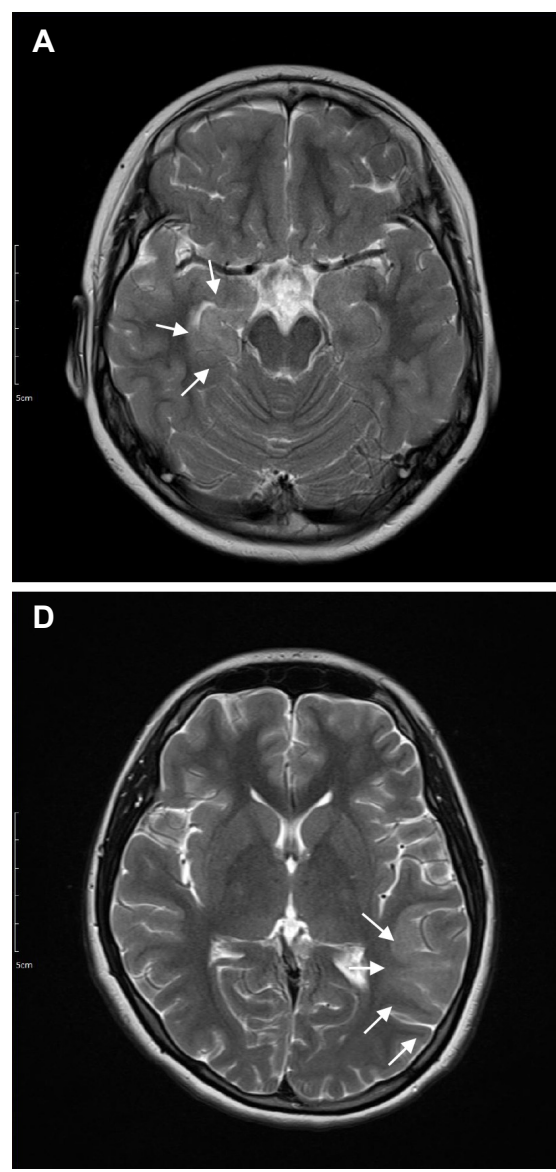

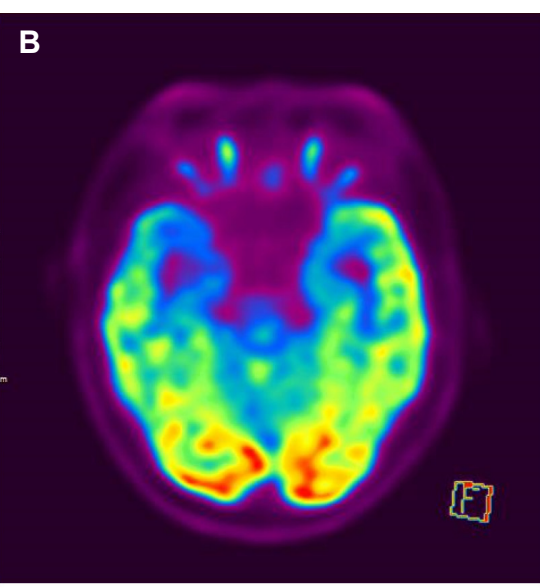

E

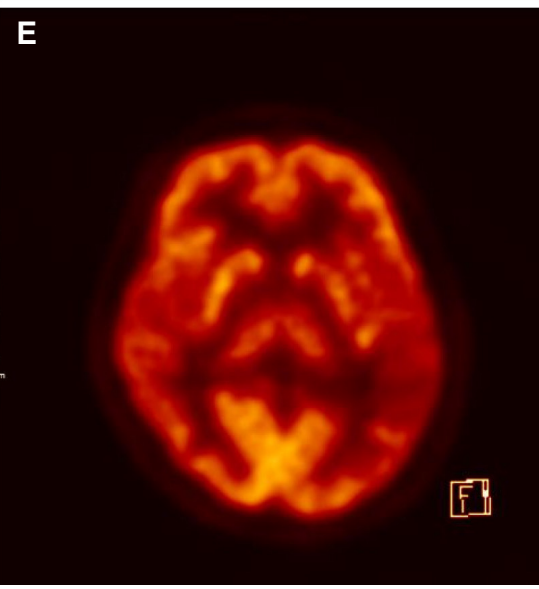

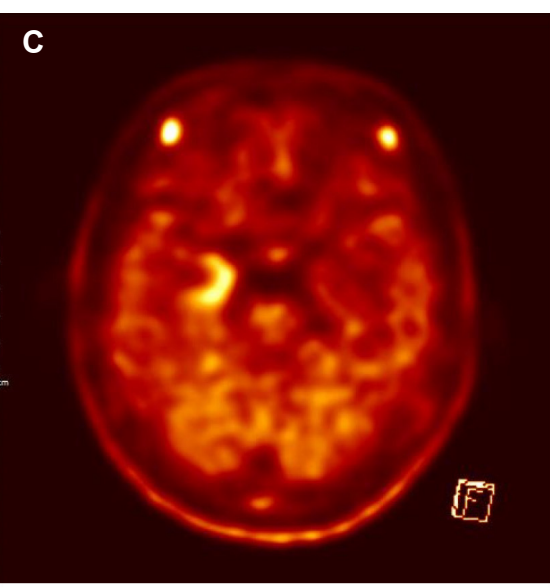

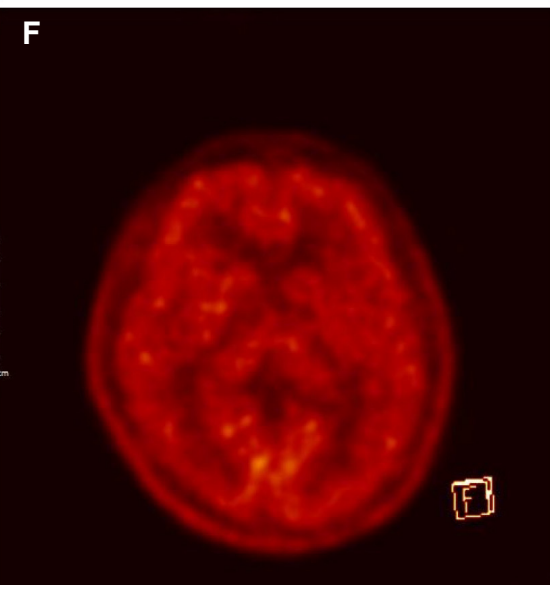

Figure 1. The difference between an MNGT and FCD on images is shown. An MR image (A, arrows) of a 12-year-old boy who had been diagnosed with a ganglioglioma after surgery shows a lesion in the right mesial temporal lobe with HSI on T2-weighted scans. The lesion is hypometabolic on ${ }^{18} \mathrm{~F}-\mathrm{FDG}$-PET images (B) but shows hot uptake on ${ }^{11} \mathrm{C}$-methionine PET images (C). On the other hand, a preoperative MR image of a 14-year-old girl with FCD type Ilb shows a T2 HSI lesion in the left posterior temporal lobe (D, arrows), and this lesion is hypometabolic on both ${ }^{18} \mathrm{~F}-\mathrm{FDG}$-PET (E) and ${ }^{11} \mathrm{C}-\mathrm{methionine}$ PET images (F). MNGT, mixed neuronal and glial tumor; FCD, focal cortical dysplasia; MR, magnetic resonance; HSI, high-signal intensity; ${ }^{18} \mathrm{~F}-\mathrm{FDG}-\mathrm{PET}$, ${ }^{18} \mathrm{~F}$-fluorodeoxyglucose positron emission tomography. 
confirmation of the lesion corresponding to the localization through electroencephalographic or invasive monitoring is essential.

${ }^{18}$ F-fluorodeoxyglucose (FDG) PET is also a commonly used imaging tool in epilepsy patients, which shows lesions as hypometabolic when obtained during the interictal period. ${ }^{22}$ It is useful to visualize the lesion itself. However, similar to high-resolution MRI, it does not distinguish between lesion types within lesional mTLE. In this case, the use of ${ }^{11} \mathrm{C}$-methionine PET is helpful for differentiating lesions. ${ }^{11} \mathrm{C}$-methionine PET results correlate well with the pathological spectrum of pediatric lesional epilepsy. ${ }^{19}$ Tumors such as DNT and gangliogliomas show a higher ${ }^{11} \mathrm{C}$-methionine uptake than $\mathrm{FCD}$. Moreover, the average lesion-to-gray matter ratio of DNTs on ${ }^{11} \mathrm{C}$-methionine PET images falls between that of FCD and gangliogliomas (Fig. 1).

Although controversy exists regarding the necessity of preoperative differential diagnosis between pathologies in lesional $m T L E$, the following considerations support the need for these pathologies to be differentiated. In the case of FCD, histological boundaries are not clear in many cases. ${ }^{23}$ Because the actual extent of the lesions may be wider than that observed on MRI, extensive epilepsy surgery is required based on the results of invasive monitoring. However, the boundaries of MNGTs are relatively clear if there is no coexisting FCD pathology, and the total resection of the tumor is known to be an important factor associated with seizure outcomes. ${ }^{8,9,23}$ The prognosis after surgery is different between these two groups; the seizure-free rates of FCD are less than $50 \%$, but seizure-free rates are approximately $80 \%$ for tumors. ${ }^{9,23,24}$ In other words, there is a difference in the determination of the surgical extent and the prediction of the surgical outcome, which suggests that it is valuable to have a way to distinguish between lesions before surgery.

The ultimate goal of epilepsy surgery is to achieve maximal seizure control without causing neurological deficits. This principle is also true for lesional mTLE. In the case of tumors, effective seizure control would be expected only by removing the entire lesion; however, in the case of FCD, this goal may not be achieved by removing the lesion as seen on images. A careful and thorough preoperative evaluation is essential to determine whether simple resection of the lesion is sufficient or if more extensive epileptic surgery is necessary after an invasive study. This requires a comprehensive discussion among a multidisciplinary team of specialists on epilepsy.

\section{Surgical Techniques}

In lesional mTLE, if selective removal of the lesion, such as selective amygdalohippocampectomy (SAH), is planned for surgery, it is crucial to approach the deep-seated lesion without removing or damaging the lateral TL. There are several selective approaches to access the mesial temporal structures, including the transsylvian, transcortical, and subtemporal approaches. In this review, the transsylvian-transcisternal approach, which is capable of sparing both lateral and basal temporal structures, ${ }^{25,26}$ is introduced briefly.

Craniotomy is performed through a conventional pterional approach. A dural incision of a semicircular shape with the base on the sphenoid is made. After exposure of the sylvian fissure, microdissection is performed from the anterior part. The chiasmatic cistern and carotid cistern are opened and dissected while draining cerebrospinal fluid, which allows the anterior temporal pole to be mobile so it can be retracted laterally. Deep into the interpeduncular cistern, the internal carotid artery and the A1 segment of the anterior cerebral artery are observed, with the optic nerve between them and the oculomotor nerve on the lateral side. Then, the sphenoid compartment of the sylvian fissure is also opened. The course of the M1 and M2 segments of the middle cerebral artery are further exposed. Deep inside, the oculomotor nerve is observed running over the arachnoid membrane. After exposing the ambient cistern and observing the P1 segment of the posterior cerebral artery, the lesion exposure dissection is completed. It is then possible to access the mesial temporal structure by turning the angle posteriorly. Lesions in mTL regions such as the amygdala, hippocampus, and parahippocampal gyrus including the uncus are accessible through this approach (Fig. 2).

The advantage of removing the selective lesion is that benign lesions may be spared to the greatest possible extent to minimize the risk of functional deficits. Hemiplegia, verbal and memory deficits, neuropsychological problems, and visual field defects are well-known complications of TLE surgery. The incidence of these complications is reported to vary from 4 to $9 \%$ in different studies. ${ }^{11,27}$ Although the positive outcomes of surgical treatments for TLE have been proven by previous studies, it is thought that concerns about such complications might prevent the widespread use of surgical treatments for TLE. ${ }^{28,29}$ In addition, considering that many lesional mTLE patients do not have neurological deficits, surgeons should strive to reduce these types of complications. Therefore, attempts have been made to reduce complications by removing only the lesions, if possible, while preserving the normally functioning 
areas.

Several studies have reported that selective surgery involves fewer complications than conventional ATL. In terms of attention, deterioration was seen in $13.3 \%$ of ATL patients whereas it was observed in only $7.1 \%$ of SAH patients, and $43.4 \%$ and $30.9 \%$ of patients showed postoperative deterioration in verbal memory, respectively. ${ }^{11}$ A study showed further difference in immediate recall in verbal memory. ${ }^{30}$ Although a meta-analysis showed no difference in verbal memory based on the surgical method, it was confirmed that there is a difference in naming outcomes based on the extent of lesion resection. ${ }^{31}$ Among the studies included in that meta-analysis, the neuropsychological assessments were conducted using different evaluation tools, which did not allow for standardization and analysis. Unfortunately, studies that show a clear contrast between the two groups are still rare. Visual field defects also appear to be less frequent in the selective approach, probably because manipulation of the roof of the temporal horn from where Meyer's loop passes is minimized. ${ }^{25}$ No comparative study has determined which selective approach is better, and a tailored approach is currently recommended for lesions. Therefore, thus far, tailored approaches based on lesions have been recommended.

One of the most important concerns in epilepsy surgery is how well seizures are controlled. Some retrospective studies have suggested that the seizure outcomes of limited resection such as SAH in TLE are not inferior to those of ATL. ${ }^{11,32}$ However, a recent meta-analysis suggested that performing ATL in TLE is better for achieving an Engel Class I outcome than is performing $\mathrm{SAH}^{33}$ Nonetheless, in cas- es of lesional mTLE, selective lesionectomy can still be considered. That is because, compared to other TLE patients, lesional mTLE patients show good postoperative seizure outcomes even when they are treated with selective lesionectomy, which is consistent with the authors' experience. ${ }^{6,25}$ Approximately $78-91 \%$ of lesional mTLE patients were classified as Engel Class I after a selective lesionectomy. One of the most important prognostic factors for good seizure outcomes is the gross total resection of the lesion. Therefore, it is necessary to evaluate in advance whether total removal is feasible. There is still lack of information specific to lesional mTLE, which requires more evidence to determine reliable seizure control rates, complication rates, and the factors affecting those rates, based on observation for longer periods of time.

It is important to resect the lesion to an extent sufficient for proper seizure control and to retain cognitive function, as well as to reduce the possibility of deterioration by minimizing damage to the functional parts of the brain. To date, it is best to devise a tailored plan depending on the patient.

\section{Conclusions}

Patients with lesional mTLE are good candidates for surgical treatment. High-resolution MRI and ${ }^{11} \mathrm{C}$-methionine PET are helpful in differentiating between tumorous and non-tumorous lesions such as FCD. It is important to determine whether a simple lesionectomy is sufficient or if more extensive epilepsy surgery is required through preoperative evaluation. In FCD, the actual lesion size is wider than
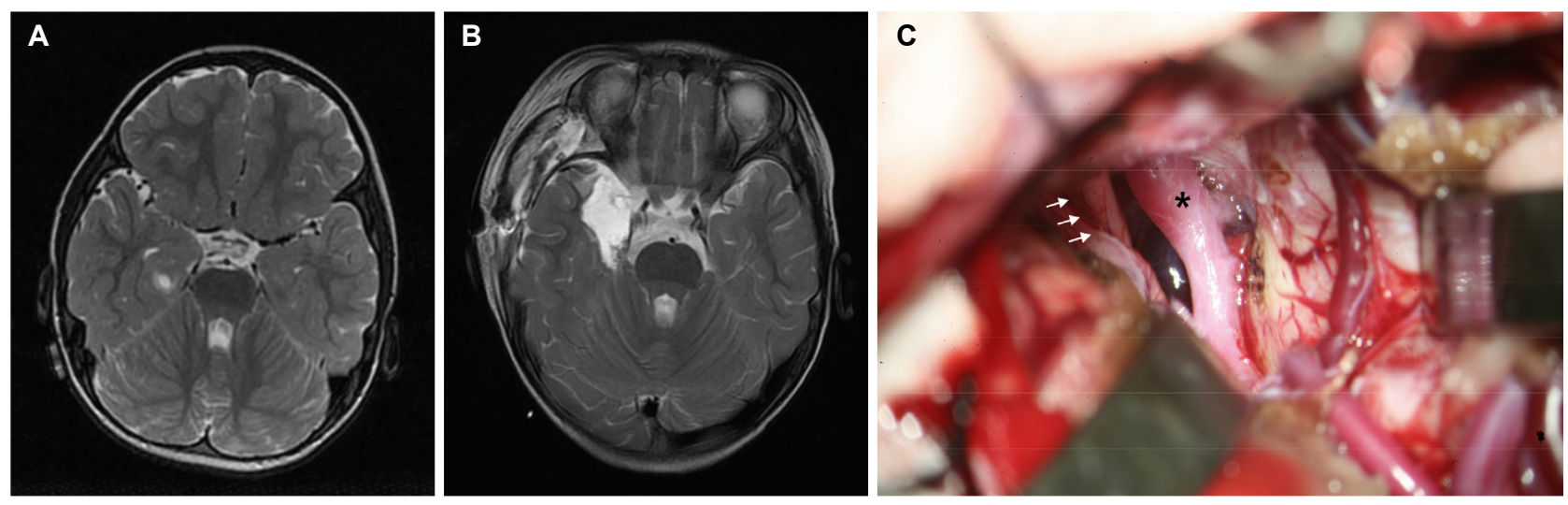

Figure 2. Preoperative (A) and postoperative (B) MR images of a 21-month-old girl who had a cystic lesion in the right mesial temporal lobe. An operative photo of the patient is shown during surgery with the transsylvian-transcisternal approach (C). The mesial temporal structures have begun to be exposed. The internal carotid artery (asterisk) and right optic nerve (arrows) are also shown. After opening the ambient cistern, more medial structures can be seen. $\mathrm{MR}$, magnetic resonance. 
that seen on the images, which may require extensive epilepsy surgery. In the case of tumorous conditions in lesional mTLE, selective lesionectomy is still considered a treatment option if total removal of the lesion is feasible. However, the presence of dual pathology along with the tumor or the presence of other epileptogenic foci should be evaluated preoperatively. In addition, the total removal of lesions should be the goal of surgery for effective control of seizures. If a selective lesionectomy is needed, the lesion can be removed safely through the transsylvian-transcisternal approach to access the mesial structures of the TL.

\section{Acknowledgements}

This research was supported by a grant from the Korea Health Technology R\&D Project through the Korea Health Industry Development Institute (KHIDI), funded by the Ministry of Health \& Welfare, Republic of Korea (grant number: HI12C0066).

\section{References}

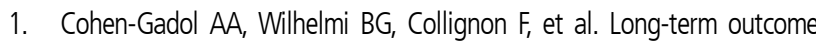
of epilepsy surgery among 399 patients with nonlesional seizure foci including mesial temporal lobe sclerosis. J Neurosurg 2006;104:513-24.

2. McIntosh AM, Kalnins RM, Mitchell LA, Fabinyi GC, Briellmann RS, Berkovic SF. Temporal lobectomy: long-term seizure outcome, late recurrence and risks for seizure recurrence. Brain 2004;127(Pt 9):2018-30.

3. Wiebe $S$, Blume WT, Girvin JP, Eliasziw M; Effectiveness and Efficiency of Surgery for Temporal Lobe Epilepsy Study Group. A randomized, controlled trial of surgery for temporal-lobe epilepsy. N Eng/ J Med 2001;345:311-8

4. Babb TL, Brown WJ. Pathological findings in epilepsy. In: Engel J Jr, ed. Surgical Treatment of the Epilepsies. New York: Raven Press, 1987:511-40.

5. Sztriha L, Gururaj AK, Bener A, Nork M. Temporal lobe epilepsy in children: etiology in a cohort with new-onset seizures. Epilepsia 2002;43:75-80.

6. Clusmann H, Kral T, Fackeldey E, et al. Lesional mesial temporal lobe epilepsy and limited resections: prognostic factors and outcome. $J$ Neurol Neurosurg Psychiatry 2004;75:1589-96.

7. Giulioni M, Galassi E, Zucchelli M, Volpi L. Seizure outcome of lesionectomy in glioneuronal tumors associated with epilepsy in children. J Neurosurg 2005;102(3 Suppl):288-93.

8. Kim SK, Wang KC, Hwang YS, Kim KJ, Cho BK. Intractable epilepsy associated with brain tumors in children: surgical modality and outcome. Childs Nerv Syst 2001;17:445-52.

9. Phi JH, Kim SK, Cho BK, et al. Long-term surgical outcomes of tem- poral lobe epilepsy associated with low-grade brain tumors. Cancer 2009;115:5771-9.

10. Zentner J, Hufnagel A, Wolf HK, et al. Surgical treatment of neoplasms associated with medically intractable epilepsy. Neurosurgery 1997;41:378-86; discussion 386-7.

11. Clusmann H, Schramm J, Kral T, et al. Prognostic factors and outcome after different types of resection for temporal lobe epilepsy. J Neurosurg 2002;97:1131-41.

12. Hill SW, Gale SD, Pearson C, Smith K. Neuropsychological outcome following minimal access subtemporal selective amygdalohippocampectomy. Seizure 2012;21:353-60.

13. Mengesha T, Abu-Ata M, Haas KF, et al. Visual field defects after selective amygdalohippocampectomy and standard temporal lobectomy. J Neuroophthalmol 2009;29:208-13.

14. Yaşargil MG, Krayenbühl N, Roth P, Hsu SP, Yasargil DC. The selective amygdalohippocampectomy for intractable temporal limbic seizures. J Neurosurg 2010;112:168-85.

15. Wurm G, Wies W, Schnizer M, Trenkler J, Holl K. Advanced surgical approach for selective amygdalohippocampectomy through neuronavigation. Neurosurgery 2000;46:1377-82; discussion 1382-3.

16. Cohen-Gadol AA, Ozduman K, Bronen RA, Kim JH, Spencer DD. Long-term outcome after epilepsy surgery for focal cortical dysplasia. J Neurosurg 2004; 101:55-65.

17. Rosenow $F$, Lüders $H$. Presurgical evaluation of epilepsy. Brain 2001;124(Pt 9):1683-700.

18. Plate $\mathrm{KH}$, Wieser HG, Yasargil MG, Wiestler OD. Neuropathological findings in 224 patients with temporal lobe epilepsy. Acta Neuropathol 1993;86:433-8.

19. Prayson RA. Tumours arising in the setting of paediatric chronic epilepsy. Pathology 2010;42:426-31.

20. Prayson RA, Reith JD, Najm IM. Mesial temporal sclerosis. A clinicopathologic study of 27 patients, including 5 with coexistent cortical dysplasia. Arch Pathol Lab Med 1996;120:532-6.

21. Raymond AA, Fish DR, Stevens JM, Cook MJ, Sisodiya SM, Shorvon SD. Association of hippocampal sclerosis with cortical dysgenesis in patients with epilepsy. Neurology 1994;44:1841-5.

22. Spencer SS. The relative contributions of MRI, SPECT, and PET imaging in epilepsy. Epilepsia 1994;35 Suppl 6:S72-89.

23. Zaatreh MM, Firlik KS, Spencer DD, Spencer SS. Temporal lobe tumoral epilepsy: characteristics and predictors of surgical outcome. Neurology 2003;61:636-41.

24. Kim DW, Lee SK, Chu K, et al. Predictors of surgical outcome and pathologic considerations in focal cortical dysplasia. Neurology 2009; 72:211-6.

25. Lee JY, Phi JH, Wang KC, Cho BK, Kim SK. Transsylvian-transcisternal selective lesionectomy for pediatric lesional mesial temporal lobe epilepsy. Neurosurgery 2011;68:582-7.

26. Vajkoczy P, Krakow K, Stodieck S, Pohlmann-Eden B, Schmiedek P. Modified approach for the selective treatment of temporal lobe epi- 
lepsy: transsylvian-transcisternal mesial en bloc resection. J Neurosurg 1998:88:855-62.

27. Hader WJ, Tellez-Zenteno J, Metcalfe A, et al. Complications of epilepsy surgery: a systematic review of focal surgical resections and invasive EEG monitoring. Epilepsia 2013;54:840-7.

28. de Flon P, Kumlien E, Reuterwall C, Mattsson P. Empirical evidence of underutilization of referrals for epilepsy surgery evaluation. Eur J Neurol 2010;17:619-25.

29. Wiebe $S$, Jetté N. Epilepsy surgery utilization: who, when, where, and why? Curr Opin Neurol 2012;25:187-93.

30. Helmstaedter C, Elger CE, Hufnagel A, Zentner J, Schramm J. Different effects of left anterior temporal lobectomy, selective amygdalohippo- campectomy, and temporal cortical lesionectomy on verbal learning, memory, and recognition. J Epilepsy 1996;9:39-45.

31. Sherman EM, Wiebe S, Fay-McClymont TB, et al. Neuropsychological outcomes after epilepsy surgery: systematic review and pooled estimates. Epilepsia 2011;52:857-69.

32. Arruda F, Cendes F, Andermann F, et al. Mesial atrophy and outcome after amygdalohippocampectomy or temporal lobe removal. Ann Neurol 1996;40:446-50.

33. Josephson $\mathrm{CB}$, Dykeman J, Fiest $\mathrm{KM}$, et al. Systematic review and meta-analysis of standard vs selective temporal lobe epilepsy surgery. Neurology 2013;80:1669-76. 